\title{
The concept of the Equivalent Length of Life for quantifying differences in age-at-death distributions across countries
}

Magdalena Muszyńska ${ }^{* *}$ and Fanny Janssen ${ }^{2,3}$

\footnotetext{
*Correspondence: mmuszyns@sgh. waw.pl

${ }^{1}$ Warsaw School of Economics, Warsaw, Poland

Full list of author information is available at the end of the article
}

\begin{abstract}
Life expectancy, that is the mean age at death in a life table, is the most common measure used to describe and compare mortality distributions. Alternatives to life expectancy that have been proposed so far have also referred to only a single parameter of the mortality distribution.

We propose to study mortality distributions by applying Silber's concept of the Equivalent Length of Life (ELL), which enables comparisons based on up to three parameters of age-at-death distributions: life expectancy, dispersion and skewness. The method, and our decomposition, is used to study convergence/divergence of life-table age-at-death distributions across 35 developed countries of the Human Mortality Database in 1970-2010 and to assess the contribution of the three moments of the distribution to the total differences between countries and trends in the contribution.

We observed a divergence of age-at-death distributions across the study countries from 1970 to 2005, followed by a convergence. Differences in life expectancies contributed the most to inequalities between the countries in life-table age-at-death distributions and the observed changes over time for both sexes. An additional important contribution resulted from the growing negative covariance between life expectancy and dispersion of ages at death, indicating that the largest increase in life expectancy occurred in the countries where variation in ages at death was lowest, especially among women. For men, including the skewness parameter resulted in lower differences between countries.

The ELL and its decomposition thus have clear added value for studying differences between countries and convergence/divergence of age-at-death distributions.
\end{abstract}

Keywords: Equivalent Length of Life, Age-at-death distributions, Mortality divergence, Mortality convergence

\section{Introduction}

Demographic studies on the determinants of mortality rely on methods of quantifying differences in mortality between regions or populations and over time. By comparing mortality across countries and over time, demographers ask how these differences are determined by unequal allocation of resources, other ecological factors or behavioural differences (Asada 2006). In the demographic literature, the majority of comparisons of population health are based on life expectancy at birth. This is the most common indicator of changes in survival over calendar time (e.g. Oeppen and Vaupel 2002;

(c) 2016 The Author(s). Open Access This article is distributed under the terms of the Creative Commons Attribution 4.0 International License (http://creativecommons.org/licenses/by/4.0/), which permits unrestricted use, distribution, and reproduction in any medium, provided you give appropriate credit to the original author(s) and the source, provide a link to the Creative Commons license, and indicate if changes were made. 
White 2002), and forms the basis for discussion of differences in population health across countries (e.g. Goesling and Firebaugh 2004; Moser et al. 2005) and across subgroups of the population, such as socio-economic disparities in mortality and health (e.g. Mackenbach et al. 1997, Mackenbach et al. 2003 or Kunst et al. 2004).

While life expectancy at birth provides a useful summary of mortality across all age groups, it refers to only one moment of mortality distributions, that is the mean age at death in a stationary population. There is a growing agreement in the demographic literature that discussion of differences in population health across various groups and over time should be based also on other measures of mortality distributions, and not only life expectancy. An example of such an additional measure is the modal age at death (the age at which most deaths occur), studied already by Lexis (1878) and more recently by Cheung et al. (2005), Canudas-Romo (2010) and Thatcher et al. (2010). There are also studies that focus on the dispersion of ages at death as an additional measure of the ageat-death distribution, using indicators such as variance, Theil and Gini indices and et, and compare their values across countries (Edwards and Tuljapurkar 2005; Tuljapurkar and Edwards 2011; Vaupel et al. 2011). Measures of inequality of ages at death are introduced into mortality studies to discuss two concepts: uncertainty of age at death and life span at the individual level (see, among others, Edwards (2013) for an extensive overview), and mortality compression (declining variability of age at dying) or expansion (increasing variability of age at dying) (i.e. Wilmoth and Horiuchi 1999).

Mostly, however, the aforementioned measures of mortality distributions are applied to compare mortality distributions across populations, once again basing on a single moment. And therefore, the problem that these distributions are generally identifiable based on a minimum of two parameters is not addressed in these studies (i.e. approximated by a normal distribution that is identified by two parameters).

To our knowledge, only four studies so far have taken into account more than one moment of the age-at-death distributions when examining differences in distributions of ages at death across a group of countries. Smits and Monden (2009) calculated Theil and Gini indices for the distribution of ages at death to study length of life inequality for the whole world. The Theil index of world inequality in ages at death was further decomposed to within- and between-country inequalities. Similarly, Edwards (2011) studied global inequality in length of life based on standard deviation, interquartile range, Gini coefficient and Theil index, and discussed the results of the decomposition of the variance and the Theil index into within- and between-country contributions to inequality in life durations. None of the two aforementioned works, however, studied differences between countries in the variation in the life span per se, since the withincountry component of the total variation is simply a sum of variations within single countries and hence does not facilitate comparisons of this statistic across countries.

Next, the study of Edwards and Tuljapurkar (2005) applied the Kullback-Leibler measure of divergence to quantify similarities in age-at-death distributions between countries and study their developments over calendar time. A limitation of this study was that the baseline distribution for comparison was that of Sweden in 2002, and it can be demonstrated that the convergence of age-at-death distributions of a country with the Swedish distribution does not necessarily guarantee the convergence of the age-at-death distributions between those countries. In the recent study by d'Albis et al. (2014), the problem of a benchmark distribution was solved by comparing the age-at- 
death distribution of each of the countries with the average for a given year. D'Albis et al. (2014) studied convergence in mortality distributions across developed countries in the years 1960-2008 by applying an index of disparity that was constructed as a sum of the results of Kullback-Leibler inequalities for single countries; total inequalities were studied separately for Eastern and for Western European countries. Unlike in the study of Edwards and Tuljapurkar (2005), however, the total convergence/divergence, as measured by the Kullback-Leibler index, was not further decomposed to study the contribution of the mean and variance of the distributions; instead they were studied and discussed separately.

The comprehensive study of distributions of ages at death across countries and over time, by means of the three moments of the distributions, is still lacking. Such a study, however, would provide us with the answer to the question what the similarities/ dissimilarities of age-at-death distributions between countries and over calendar time constitute of, or phrased differently, what is the contribution of differences in life expectancy, differences in dispersion of ages at death and differences in asymmetry of the distribution to the between-country variation and convergence/divergence of the distributions over time. To answer these questions, the development of a new method is essential.

In the present study, we propose to use the concept of the Equivalent Length of Life (ELL) by Silber (1983) to comprehensively compare distributions of ages at death across countries and over time. Apart from having a meaningful demographic interpretation, as "...length of life which, if being identical for all individuals, would give the same social welfare as the actual distribution of deaths by age." (Silber 1983, p.21), the ELL summarizes mortality distributions in a single indicator but takes into account up to three moments: mean, variance and skewness. We also demonstrate that the total variation between countries in age-at-death distributions, quantified by the variance of the ELL, can be easily decomposed into the contribution of differences between the means, standard deviations and skewness parameters of the age-at-death distribution in individual countries. Hence, by applying the concept of the ELL a full picture of similarities/dissimilarities of age-at-death distributions between countries and over calendar time can be obtained. Moreover, for the first time, it will be possible to assess the contribution of differences in life expectancy, differences in dispersion of ages at death and in asymmetry to the between-country variation and convergence/divergence of the distributions over time.

In the empirical part of the study, we demonstrate the added value of the proposed method by studying convergence/divergence of life-table age-at-death distributions across the countries of the Human Mortality Database (2014) in the years 1970-2010, and assess the contribution of the three moments of the distribution to the total differences between countries and its trends over the study years.

\section{Method}

\section{The concept of Equivalent Length of Life}

The concept of the Equivalent Length of Life (ELL) was introduced by Silber (1983) as a development indicator, based on concepts by Atkinson (1970) and Kolm (1976a, b), and measures "...length of life which, if being identical for all individuals, would give 
the same social welfare as the actual distribution of deaths by age." (Silber 1983, p.21). Thus, the formula for the ELL is derived from the Social Welfare Function concept as:

$$
\mathrm{ELL}=e_{0}(1-I)
$$

where $I$ stands for any inequality index and $e_{0}$ for life expectancy. Please note that this formula, and similarly the subsequent formulas, can be applied to different times and different countries. For simplicity reasons, we however did not add subscripts to the formulas.

When writing the formula for the ELL, one has to decide on a scale invariance of $I$ : that is, the response of the statistic to proportional and absolute equal change in lifetime durations in the life table. In this paper, we focus on measures based on variance, and therefore in the above formula, we apply the coefficient of variation as the inequality index. According to Silber (1983), one more choice needs to be made concerning the statistic under study. One should decide whether to give equal weight to differences in length of life at all ages, or whether the weights are different, for example for younger age groups. As our main research question concerns differences in the distributions of ages at death and we do not intend to place any normative judgment on those differences, we decided to give equal weight to deaths at all ages under study.

When the coefficient of variation is employed as an inequality measure, ELL reduces to:

$$
e_{C}=e_{0}\left(1-\frac{\mathrm{SD}}{e_{0}}\right)=e_{0}-\mathrm{SD}
$$

where SD stands for the standard deviation, or disparity, of life-table ages at death. As a result, the new index, $e_{C}$, where the mean duration of life $e_{0}$ is discounted for the dispersion of ages at death, enables comparisons between populations that take into account both life expectancy and disparity of ages at death.

Furthermore, following Silber (1988), we take into account asymmetry of the distribution of ages at death in a modified index $e_{A}$, defined in this case as:

$$
e_{A}=e_{0}-\mathrm{SD}(1-A)
$$

where $A$ is a measure of asymmetry. Hence, $e_{A}$ adjusts life expectancy for both dispersion and skewness of the age-at-death distribution.

As the asymmetry measure $A$, we use the relative Asymmetry Index proposed by Berrebi and Silber (1987) and then applied to the modified ELL by Silber (1988):

$$
A=\frac{1}{2} \frac{\mathrm{SD}_{U}-\mathrm{SD}_{L}}{\mathrm{SD}}
$$

where $\mathrm{SD}$ is the standard deviation of ages at death of the whole distribution, $\mathrm{SD}_{U}$ is the standard deviation of ages at death of those who live longer than the median age at death and $\mathrm{SD}_{L}$ stands for the standard deviation in ages at death of those who live fewer years than the median age at death.

By adjusting the index of $e_{C}$ also for asymmetry of the distributions, we add information on the quantile of the distribution at which the dispersion mostly occurs. As a result, with relatively stable variation of ages at death for short-lived individuals in developed countries, the inclusion of the skewness parameter in the ELL summary measure means adding information about the level of variability of mortality among the longest-lived individuals. In practice, the adjustment for asymmetry of the distribution applies to the element of the ELL that refers to uncertainty of ages of death. In 
developed countries, the uncertainty attributed to inequality of ages at death occurs mostly for the shortest-lived $50 \%$ of individuals, $\mathrm{SD}_{L}>\mathrm{SD}_{U}$ and the distribution is skewed to the left. Given the same level of variation for short-lived individuals, the lower the variability or the higher the compression of ages at death among long-lived individuals, the lower the value of $\mathrm{SD}_{U}$ and the more left-skewed the distribution, resulting in a smaller correction of life expectancy for the uncertainty in the ELL summary measure. As a result, the more uncertainty is concentrated among the longestlived individuals, the closer the value of $e_{A}$ is to $e_{0}$.

\section{Decomposition}

Differences in the value of the ELL across developed countries can be quantified with variance $\left(S^{2}\right)$. In addition, the total variance of the ELL distribution (either $e_{C}$ or $e_{A}$ ) can be decomposed into the variance of its components and their covariance.

For $e_{C}$, the total variance can be decomposed into its components (life expectancy and disparity of ages at death) and their covariance, according to a formula that is derived from a simple decomposition of the variance of the sum of two variables:

$$
S^{2}\left(e_{C}\right)=S^{2}\left(e_{0}\right)+S^{2}(\mathrm{SD})-2 \operatorname{cov}\left(e_{0}, \mathrm{SD}\right)
$$

The first term stands for differences in life expectancies as measured by the variation in $e_{0}$ and the second term stands for differences in dispersion parameters as measured by the variation in $\mathrm{SD}$. The third term indicates the covariance between $e_{0}$ and $\mathrm{SD}$ across the group of countries under study. For example, negative covariance would indicate that countries with high life expectancy are also characterized by low dispersion of ages at death. In a study of changes over time in the variance in $e_{C}$ in a group of countries, the contribution of changes in life expectancy and of changes in the dispersion of ages at death (compression when dispersion is declining, expansion when dispersion is increasing) can be assessed accordingly.

When differences in the value of $e_{A}$ across developed countries are quantified with variance, $e_{A}$ reduces to:

$$
e_{A}=e_{0}-\mathrm{SD}+A^{\prime}
$$

where $A^{\prime}=\frac{1}{2}\left(\mathrm{SD}_{U}-\mathrm{SD}_{L}\right)$.

Variance in $e_{A}$ can be decomposed into the following elements:

$$
S^{2}\left(e_{A}\right)=S^{2}\left(e_{0}\right)+S^{2}(\mathrm{SD})+S^{2}\left(A^{\prime}\right)-2 \operatorname{cov}\left(e_{0}, \mathrm{SD}\right)-2 \operatorname{cov}\left(\mathrm{SD}, A^{\prime}\right)+2 \operatorname{cov}\left(e_{0}, A^{\prime}\right)
$$

The first three elements stand for variance in the three parameters of the distribution (life expectancy, disparity, skewness), and the next three elements indicate covariance between each pair of the parameters. The three covariance elements may be used to answer additional important research questions. Is high life expectancy achieved at low disparity of ages at death, because all individuals are long-lived, or does the high life expectancy result from high skewedness, that is extended life durations of a small group of long-lived individuals? Does high dispersion of ages at death result from large differences (=decompression) of mortality at old age, or large differences among shortlived individuals? And, when applied to analysis of data over time, what is the relationship between increases in life expectancy and less dispersion (=compression) of mortality among long-lived individuals? 


\section{Empirical application}

The methods proposed above are applied to study differences in mortality and age-atdeath distributions, and their convergence/divergence in developed countries over the years 1970-2010, separately for males and females. Data used in the empirical part of the study come from the Human Mortality Database (2014). Out of the 38 countries present in the database, Chile, Israel and Slovenia were excluded due to the short time period covered by the database for those countries. For the remaining 35, we estimated the statistics in the common period covered, that is between 1970 and 2010 (or the last year available, but not earlier than 2008).

As was the case in the previous studies of differences in age-at-death distributions (Edwards 2011; Edwards and Tuljapurkar 2005; Smits and Monden 2009), we eliminated differences in infant and childhood mortality, studying truncated distributions of the length of life above the age of completed 10 years. From life tables of individual countries, we estimated the value of the expected number of years lived for those aged $10\left(e_{10}\right)$; the other statistics for the Equivalent Length of Life $\left(e_{C}\right.$ and $\left.e_{A}\right)$ are also based on this limited age range.

\section{Empirical results}

In 2010, the average life expectancy at age $10\left(e_{10}\right)$ for women in the 35 selected countries was 71.6 years, with an average dispersion of the life-table age-at-death distribution (SD) of 12.6 years, resulting in an average $e_{C}$ of 59.0 years, and an average skewness of the lifetable age-at-death distribution $(A)$ of -0.29 , resulting in an average $e_{A}$ of 55.3 (Table 1). For women, the variance $\left(S^{2}\right)$ across the 35 countries proved to be the highest for the ageat-death statistic $e_{A}$ (11.8), that is when taking into account all the three moments of the age-at-death distribution (mean, dispersion, skewness). Compared to women, the average $e_{10}$ for men in 2010 is lower (65.3), but dispersion of ages of death (SD) is higher (14.3), leading to a lower average $e_{C}$ (51.0) and $e_{A}$ (47.5) (Table 1). The variance between the countries in the summary measures of age-at-death distributions is much higher for men than women. The highest variance for men is observed for $e_{c}$ (34.2).

For both men and women, the covariance between $e_{10}$ and SD in 2010 was negative, indicating that countries with higher life expectancy were also characterized by lower dispersion of ages at death. The relationship between skewness and dispersion on the one hand and skewness and the mean age at death $\left(e_{10}\right)$ on the other hand depends on sex.

From 1970 to 2005, a divergence between the selected countries in age-at-death distributions was observed, indicated by an increase in the variance of the three summary measures $\left(e_{10}, e_{C}, e_{A}\right)$ for both sexes, followed by a convergence (=declining variance) in the distributions between 2005 and 2010 (Table 1 and Fig. 1).

The smallest change in variance in both periods was observed for the measure based only on the mean value, that is the expected number of years lived at age $10\left(e_{10}\right)$. The largest increase in variance between 1970 and 2005 occured for women when the expected number of years lived were adjusted for both dispersion and skewness $\left(e_{A}\right)$, whereas for men this occured for $e_{C}$, which only involves adjustment for dispersion. The highest decline in variance over the next 5 years occurred in the expected number of years lived adjusted only for dispersion $\left(e_{C}\right)$. Thus, not only did divergence and subsequent convergence occur in the mean value of the age-at-death distribution, this process was even more pronounced when distributions were additionally summarized by their dispersion 
Table 1 Mean and variance $\left(S^{2}\right)$ of the summary measures of the life-table age-at-death distributions $\left(e_{10}, e_{C}, e_{A}\right)$ and mean, variance and covariance of its components (dispersion SD, and skewness $A^{\prime}$ ) across 35 developed countries, by sex. Selected years between 1970 and 2010, and absolute change in the statistics in two sub-periods: 1970-2005 and 2005-2010

\begin{tabular}{|c|c|c|c|c|c|c|c|c|}
\hline Statistic & 1970 & 1980 & 1990 & 2000 & 2005 & $2010^{a}$ & 1970-2005 & $2005-2010$ \\
\hline \multicolumn{9}{|l|}{ Women } \\
\hline $\operatorname{Mean}\left(e_{10}\right)$ & 65.7 & 67.0 & 68.3 & 69.7 & 70.6 & 71.6 & 4.9 & 1.0 \\
\hline Mean $\left(e_{C}\right)$ & 52.4 & 53.8 & 55.2 & 56.8 & 57.8 & 59.0 & 5.4 & 1.2 \\
\hline $\operatorname{Mean}\left(e_{A}\right)$ & 48.9 & 50.3 & 51.6 & 53.1 & 54.2 & 55.3 & 5.3 & 1.1 \\
\hline Mean(SD) & 13.3 & 13.2 & 13.1 & 12.9 & 12.7 & 12.6 & -0.7 & -0.1 \\
\hline Mean $(A)$ & -0.27 & -0.27 & -0.27 & -0.28 & -0.29 & -0.29 & -0.02 & 0.00 \\
\hline$S^{2}\left(e_{10}\right)$ & 1.43 & 3.04 & 4.49 & 7.44 & 8.94 & 7.37 & 7.51 & -1.57 \\
\hline$s^{2}\left(e_{c}\right)$ & 2.04 & 3.99 & 5.95 & 10.80 & 13.83 & 11.34 & 11.79 & -2.49 \\
\hline$S^{2}\left(e_{A}\right)$ & 2.19 & 4.09 & 5.66 & 10.82 & 14.22 & 11.76 & 12.04 & -2.46 \\
\hline$S^{2}(S D)$ & 0.43 & 0.41 & 0.30 & 0.50 & 0.74 & 0.63 & 0.31 & -0.11 \\
\hline$S^{2}\left(A^{\prime}\right)$ & 0.04 & 0.07 & 0.06 & 0.08 & 0.07 & 0.07 & 0.03 & 0.00 \\
\hline $\operatorname{cor}\left(e_{10}, \mathrm{SD}\right)$ & -0.11 & -0.25 & -0.50 & -0.74 & -0.80 & -0.77 & -0.69 & 0.03 \\
\hline $\operatorname{cor}\left(S D, A^{\prime}\right)$ & -0.54 & -0.38 & -0.06 & -0.31 & -0.49 & -0.48 & 0.05 & 0.01 \\
\hline $\operatorname{cor}\left(e_{10}, A^{\prime}\right)$ & -0.11 & -0.10 & -0.36 & -0.11 & 0.06 & 0.11 & 0.16 & 0.05 \\
\hline \multicolumn{9}{|l|}{ Men } \\
\hline $\operatorname{Mean}\left(e_{10}\right)$ & 59.5 & 60.0 & 61.2 & 62.7 & 63.8 & 65.3 & 4.3 & 1.5 \\
\hline Mean $\left(e_{C}\right)$ & 44.5 & 45.1 & 46.3 & 48.0 & 49.4 & 51.0 & 4.9 & 1.6 \\
\hline $\operatorname{Mean}\left(e_{A}\right)$ & 41.0 & 41.7 & 42.8 & 44.5 & 46.0 & 47.5 & 5.0 & 1.5 \\
\hline Mean(SD) & 15.0 & 14.9 & 14.9 & 14.7 & 14.4 & 14.3 & -0.6 & -0.1 \\
\hline Mean $(A)$ & -0.23 & -0.23 & -0.23 & -0.24 & -0.24 & -0.25 & -0.01 & -0.01 \\
\hline$S^{2}\left(e_{10}\right)$ & 3.11 & 7.22 & 11.13 & 22.58 & 29.28 & 23.27 & 26.17 & -6.01 \\
\hline$S^{2}\left(e_{C}\right)$ & 5.89 & 12.61 & 16.96 & 32.95 & 42.28 & 34.17 & 36.39 & -8.21 \\
\hline$S^{2}\left(e_{A}\right)$ & 6.37 & 12.22 & 15.34 & 27.16 & 34.55 & 28.46 & 28.18 & -6.09 \\
\hline$S^{2}(S D)$ & 4.97 & 5.45 & 4.94 & 5.48 & 7.72 & 7.10 & 2.75 & -0.62 \\
\hline$S^{2}\left(A^{\prime}\right)$ & 0.16 & 0.15 & 0.25 & 0.46 & 0.93 & 0.86 & 0.77 & -0.07 \\
\hline $\operatorname{cor}\left(e_{10,}, \mathrm{SD}\right)$ & -0.74 & -1.03 & -1.39 & -3.47 & -7.35 & -6.56 & -6.61 & 0.78 \\
\hline $\operatorname{cor}\left(S D, A^{\prime}\right)$ & $-0,60$ & -0.16 & -0.04 & 0.52 & 0.61 & 0.58 & 1.21 & -0.03 \\
\hline $\operatorname{cor}\left(e_{10}, A^{\prime}\right)$ & 0.03 & -0.36 & -0.59 & -0.86 & -0.90 & -0.86 & -0.93 & 0.04 \\
\hline
\end{tabular}

Notes: the table shows variance in statistics of the distribution of life-table ages at death above age $10 ; e_{10}$ is the life expectancy at age $10 ; e_{C}$ is the Equivalent Length of Life with $e_{10}$ adjusted for dispersion of ages at death above age 10; $e_{A}$ is the Equivalent Length of Life with $e_{10}$ adjusted for dispersion and skewness of ages at death above age 10; SD is the standard deviation; $A$ and $A^{\prime}$ are the skewness statistics; cor stands for correlation. The statistics of the life-table age-at-death distribution above age 10 for the individual countries, by sex, and selected years, can be viewed from the Appendix (Table 3 and Fig. 2). Source: Authors' estimations based on Human Mortality Database (2014) ${ }^{\mathrm{a} O r}$ latest available year

and skewness. For HMD populations, we thus observe both divergence followed by convergence in the shape of the age-at-death distribution.

Interestingly, while for women differences between the variances in the three measures are small and the values of $e_{C}$ and $e_{A}$ are almost equal, for men they are large and grow over the study years. In general, distributions of ages at death for single countries are characterized by significantly bigger differences in the three moments of the distribution for males than for females. These differences are to a large extent the result of inclusion of Eastern European countries where, for men, substantially different values are observed than in the remaining countries: lower life expectancy, higher inequality of ages at death 


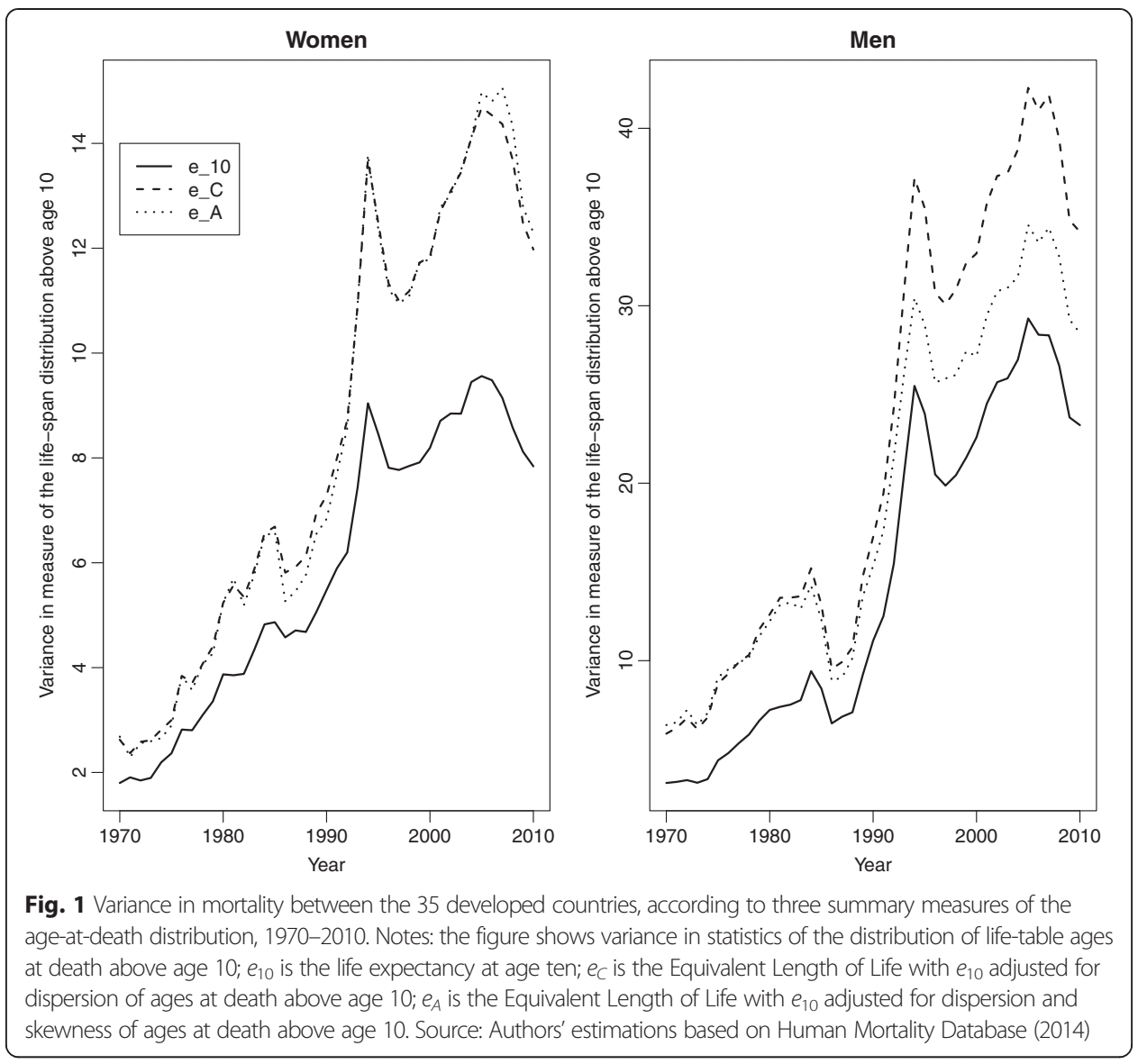

and lower negative skewness of the distributions (Appendix: Table 3 and Fig. 2). The low mean and relatively high variance of ages at death in Eastern Europe have been discussed in detail by D'Albis et al. (2014). The lower negative skewness we observed for Eastern European countries results mainly from a lower variability, or compression, of ages at death among the longest-lived $50 \%$ of individuals than in the remaining countries.

Table 2 shows the results of decomposing the total variance in $e_{C}$ and $e_{A}$ between the countries into their components. For both sexes, between-country differences in the mean age at death were the largest factor responsible for differences in the age-at-death distributions. That is, variance in the exped number of years lived $\left(e_{10}\right)$ was the largest contributor to the variance in the two measures of the ELL: $e_{C}$ and $e_{A}$. While for women the contribution of differences between mean values to the total variation decreased from 1990 onwards, for men, we observe an increase of this contribution over the study period.

The next factor important for the differences in the summary measures was the gap between countries with both high life expectancy and low dispersion of ages at death (e.g. Northern European countries) versus countries with both low life expectancy and high dispersion (e.g. Eastern Europe). That is, the second largest contribution to the total variance in $e_{C}$ and $e_{A}$ was of the covariance between $e_{10}$ and the standard deviation of ages at death (SD). As may be seen in Table 1 , the covariance between $e_{10}$ and SD was itself negative, which indicates that countries with higher life expectancy were also characterized by lower dispersion of ages at death. The gap between countries with higher life expectancy and lower dispersion of ages at death, and the countries with lower life expectancy and higher dispersion grew over the years 1970-2005, as indicated by the increasing negative correlation between the two measures shown in Table 1. These growing differences between 
Table 2 Contribution (in percent) to the variance in the ELL summary measures $e_{C}$ and $e_{A}$ across 35 developed countries of the different components constituting the summary measures (dispersion SD and skewness $A^{\prime}$, and their interrelations), by sex, and for selected years from 1970 to 2010

\begin{tabular}{|c|c|c|c|c|c|c|}
\hline Statistic & 1970 & 1980 & 1990 & 2000 & 2005 & $2010^{a}$ \\
\hline & \multicolumn{6}{|c|}{ Women } \\
\hline & \multicolumn{6}{|c|}{ Contribution to the variance in $e_{C}$} \\
\hline$S^{2}\left(e_{10}\right)$ & 0.70 & 0.76 & 0.75 & 0.69 & 0.65 & 0.65 \\
\hline$S^{2}(S D)$ & 0.21 & 0.10 & 0.05 & 0.05 & 0.05 & 0.06 \\
\hline \multirow[t]{2}{*}{$-2 \operatorname{cov}\left(e_{10,}, \mathrm{SD}\right)$} & 0.09 & 0.14 & 0.20 & 0.27 & 0.30 & 0.29 \\
\hline & \multicolumn{6}{|c|}{ Contribution to the variance in $e_{A}$} \\
\hline$S^{2}\left(e_{10}\right)$ & 0.66 & 0.74 & 0.79 & 0.69 & 0.63 & 0.63 \\
\hline$S^{2}(S D)$ & 0.20 & 0.10 & 0.05 & 0.05 & 0.05 & 0.05 \\
\hline$S^{2}\left(A^{\prime}\right)$ & 0.02 & 0.02 & 0.01 & 0.01 & 0.00 & 0.01 \\
\hline$-2 \operatorname{cov}\left(e_{10,}, S D\right)$ & 0.08 & 0.13 & 0.21 & 0.26 & 0.29 & 0.28 \\
\hline$-2 \operatorname{cov}\left(S D, A^{\prime}\right)$ & 0.10 & 0.04 & 0.00 & 0.02 & 0.02 & 0.03 \\
\hline \multirow[t]{3}{*}{$2 \operatorname{cov}\left(e_{10}, A^{\prime}\right)$} & -0.02 & -0.02 & -0.06 & -0.01 & 0.01 & 0.01 \\
\hline & \multicolumn{6}{|l|}{ Men } \\
\hline & \multicolumn{6}{|c|}{ Contribution to the variance in $e_{C}$} \\
\hline$S^{2}\left(e_{10}\right)$ & 0.53 & 0.57 & 0.66 & 0.69 & 0.69 & 0.68 \\
\hline$S^{2}(S D)$ & 0.16 & 0.10 & 0.06 & 0.04 & 0.04 & 0.04 \\
\hline \multirow[t]{2}{*}{$-2 \operatorname{cov}\left(e_{10}, \mathrm{SD}\right)$} & 0.31 & 0.33 & 0.28 & 0.28 & 0.27 & 0.28 \\
\hline & \multicolumn{6}{|c|}{ Contribution to the variance in $e_{A}$} \\
\hline$S^{2}\left(e_{10}\right)$ & 0.49 & 0.59 & 0.73 & 0.83 & 0.85 & 0.82 \\
\hline$S^{2}(S D)$ & 0.15 & 0.10 & 0.07 & 0.05 & 0.04 & 0.05 \\
\hline$S^{2}\left(A^{\prime}\right)$ & 0.01 & 0.01 & 0.01 & 0.02 & 0.02 & 0.01 \\
\hline$-2 \operatorname{cov}\left(e_{10}, S D\right)$ & 0.29 & 0.34 & 0.31 & 0.33 & 0.33 & 0.34 \\
\hline$-2 \operatorname{cov}\left(S D, A^{\prime}\right)$ & 0.12 & 0.02 & 0.00 & -0.03 & -0.04 & -0.04 \\
\hline $2 \operatorname{cov}\left(e_{10}, A^{\prime}\right)$ & 0.01 & -0.05 & -0.12 & -0.20 & -0.21 & -0.18 \\
\hline
\end{tabular}

Notes: the table shows variance in statistics of the distribution of life-table ages at death above age 10; $e_{10}$ is the life expectancy at age 10; $e_{C}$ is the Equivalent Length of Life with $e_{10}$ adjusted for dispersion of ages at death above age 10; $e_{A}$ is the Equivalent Length of Life with $e_{10}$ adjusted for dispersion and skewness of ages at death above age 10; SD is the standard deviation; $A^{\prime}$ is the skewness statistic; cov is covariance. Source: Authors' estimations based on Human Mortality Database (2014)

${ }^{\mathrm{a}}$ Or latest available year

countries resulted in an increasing relative contribution of this factor to the total variation in $e_{C}$ for women, but for men its relative contribution to $e_{C}$ remained comparatively stable despite an absolute level that was times higher.

At the same time, differences between countries in the age-at-death dispersion itself, as measured by SD, were small and of little and decreasing importance for the total differences between distributions. Similarly, the contribution of the variance in the skewness statistic $\left(A^{\prime}\right)$ to the total differences in $e_{A}$ was small. For men, a large effect of asymmetry on the variance in $e_{A}$ was present since 1980 in the form of negative contribution of the covariance between $e_{0}$ and $A^{\prime}$ to the variance in $e_{A}$. The relationship between the two measures was negative for males (Table 1) and increased until 2005. For women, the contribution of this element is negligible.

For men after 1980, the negative and high contributions of the covariance between mean and inequality as well as between mean and skewness to the differences between countries result from the existence of outliers (Eastern European countries), as 
discussed above. The gap between age-at-death distributions of the Eastern European countries and the remaining countries also grew for males over the study years and hence resulted in the growing contribution of the two elements to the total inequality.

For women, starting in the 1980s, only the covariance between mean and inequality was of importance to the total disparities in $e_{C}$ and $e_{A}$. Like for men, this was the effect of outliers, that is Eastern European countries, which have relatively low mean and high dispersion of ages at death (Appendix: Table 3 and Fig. 2). Unlike for males, distributions for women in these countries are characterized by a high level of negative skewness among the study group. Hence for women, Eastern European countries are characterized by a high compression of mortality for the longest-lived $50 \%$ of individuals and most of the uncertainty of ages at death occurs for the short-lived individuals. The remaining female populations are characterized by relatively higher life expectancies, lower dispersion of ages of death, but also higher decompression (=expansion) of mortality for long-lived individuals.

\section{Discussion}

We demonstrated the usefulness of a set of measures for quantifying differences across countries in age-at-death distributions based on Silber's (1983) Index of Equivalent Length of Life (ELL). Applying the concept of the ELL allows life-table distributions of ages at death in individual countries to be quantified with a summary measure that takes into account, in addition to the mean (that is life expectancy), also the dispersion and skewness of the distributions. To measure the latter two, we applied indicators invariant to absolute equal change in the mortality distributions that are derived from the variance. Moreover, we demonstrated that differences between countries in the ELL-based summary measures can be quantified using variance, which is easily decomposed into the contribution of the differences in the mean, dispersion and asymmetry of the distribution of ages at death. Although other measures of disparity may be applied to derive inequalities of ages at death in life tables and between-country differences in the summary indices, we chose to use variance due to its aforementioned relatively easy decomposition into the contribution of the parameters to the total variation between countries.

Our empirical application of the proposed measures to mortality in 35 developed countries over the years 1970-2010 showed, for both sexes and independent of the three summary measures $\left(e_{10}, e_{C}, e_{A}\right)$ of the age-at-death distributions applied, a divergence between countries in age-at-death distributions between 1970 and 2005, followed by a convergence in the years 2005-2010. While the divergence of distributions between 1970 and 2005 was also reported in the study of d'Albis et al. (2014), the convergence after 2005, despite being present in the figures, was not reported by the authors. It could well be that because the study period ended in 2008, the authors regarded the post-2005 trend as a random fluctuation from the diverging trend. However, our analysis clearly shows a trend reversal from divergence to convergence, which could reflect a new phase in the mortality transition.

For both sexes, between-country differences in the age-at-death distributions and their changes over time were the largest when both additional parameters of the distributions (after life expectancy) were taken into account in the summary indices of the ELL. While for women the variance in $e_{A}$ and $e_{C}$ showed fairly similar levels over time, for men, the two statistics varied significantly. Differences between the sexes in the compression/expansion of mortality have been observed before (e.g. Janssen, Rousson, Paccaud 2015). Our results clearly 
suggest that for men it is actually essential to consider both dispersion and skewness in the summary measure of the age-at-death distributions when examining differences between countries.

When age-at-death distributions were summarized with the ELL (either by $e_{C}$ or $e_{A}$ ) and hence included more than one moment of the distribution, both differences in the mean and in the dispersion of life-table ages at death turned out to be important for the total variation between countries. Also the negative correlation between the two statistics proved important for the differences in the summary measures. This negative correlation between the two parameters, which indicates that countries with higher life expectancy were also characterized by lower dispersion of ages at death, was previously reported by Vaupel et al. (2011) and d'Albis et al. (2014).

Furthermore, the negative and high contribution of the covariance between mean and inequality and between mean and skewness to the differences between countries, observed for men since 1980, results from the existence of outliers. These outliers are countries of Eastern Europe, characterized by low mean, high dispersion and low skewness of ages at death. Ignoring skewness as one of the parameters summarizing the age-at-death distribution for males would therefore result in an underestimation of between-country differences among men in the countries and periods studied. For women, from the 1980s, only the covariance between mean and inequality was of importance to the total disparities in $e_{C}$ and $e_{A}$. Like for men, for women, this is the effect of outliers, that is Eastern European countries, with relatively low mean and high dispersion of ages at death. Unlike for males, distributions for women in these countries are characterized by a high level of negative skewness among the study group. Hence, women in Eastern European countries are characterized by a high compression of mortality for the longest-lived $50 \%$ of individuals and most of the uncertainty of ages at death occurs for the short-lived individuals. Women in the remaining countries have relatively higher life expectancies, lower dispersion of ages of death, but also higher expansion of mortality for long-lived individuals. The above clearly warrants the use of $e_{A}$ instead of $e_{C}$ to summarize variation between countries in the age-at-death distribution.

\section{Conclusions}

The empirical part of the study demonstrated that trends in the variation in the three summary measures of age-at-death distributions $\left(e_{10}, e_{C}, e_{A}\right)$ follow the same pattern. In addition, in the study of differences between distributions and their convergence/divergence over calendar time, the largest contributing factor is changes in between-country variation in life expectancies. These results might suggest that it is sufficient to summarize distributions in this type of studies with a single parameter, that is life expectancy. However, additional insights emerged from the comparison of variation in life expectancy and the two summary measures that take into account dispersion and asymmetry of the distribution. The empirical study showed that the covariance between life expectancy and the dispersion of ages at death contributed substantially to total differences between countries and to their convergence. Furthermore, for men, an additional effect of the symmetry of the age-atdeath distribution showed, warranting the use of the ELL-type index that also includes the skewness parameter. The proposed measures, based on the ELL, and the decomposition thus generate additional conclusions on the importance of the three parameters of age-atdeath distributions for total differences between countries, and reveal the true extent of these differences. 


\section{Appendix}

Table 3 Parameters of the life-table age-at-death distributions and the summary measures $\left(e_{10}, e_{C}, e_{A}\right)$ in 35 developed countries, by sex, 2010

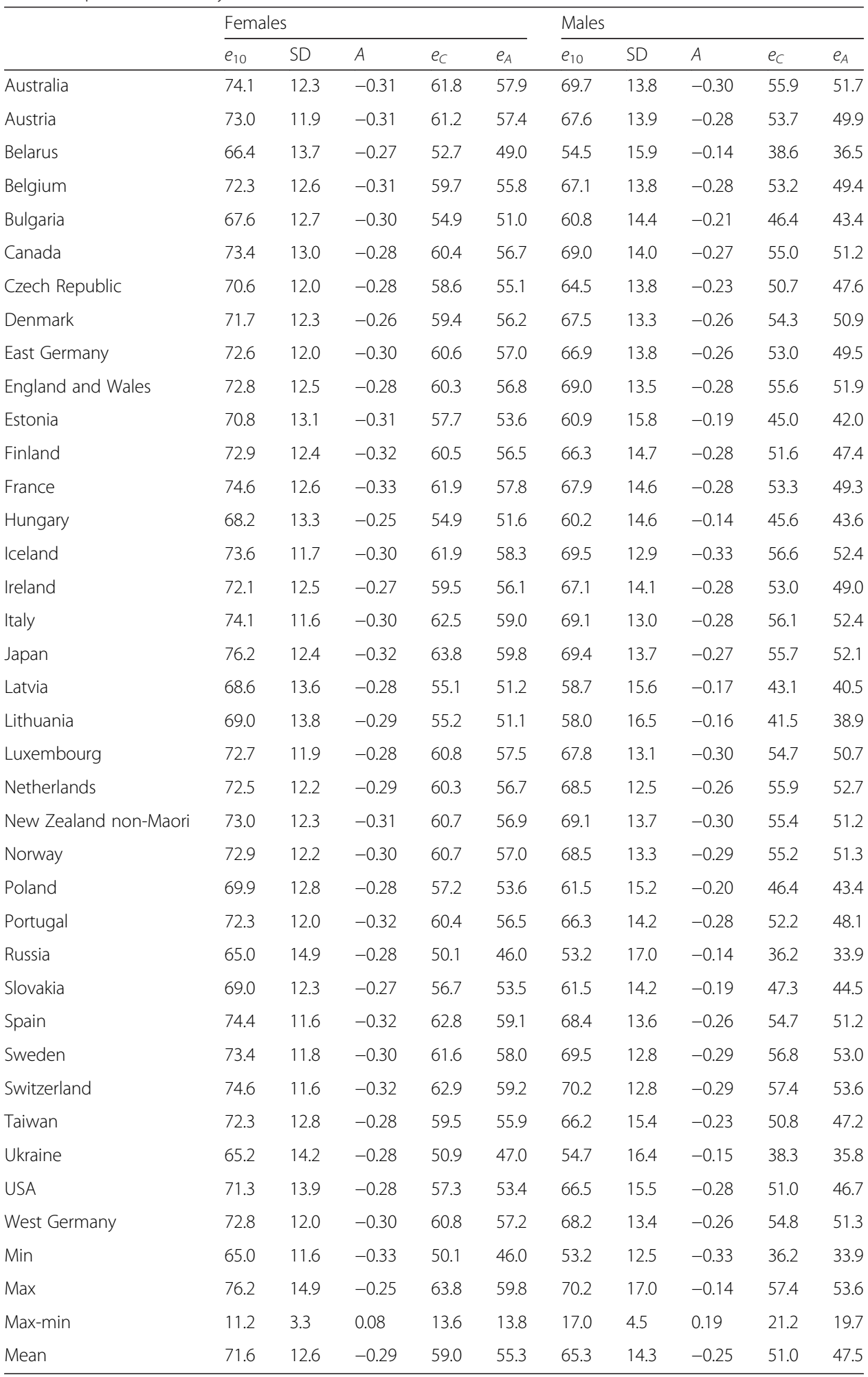

Source: Authors' estimations based on Human Mortality Database (2014) 


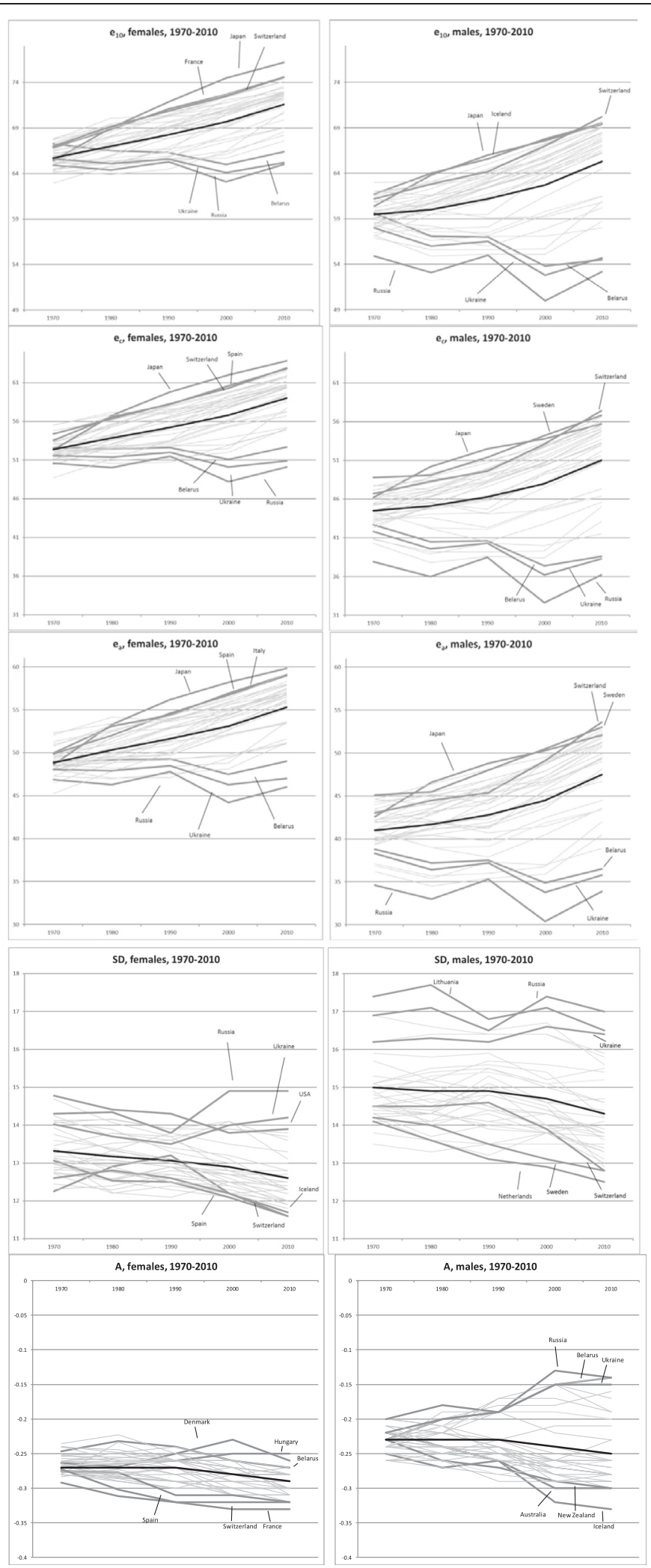

Fig. 2 Parameters of the life-table age-at-death distributions and the summary measures $\left(e_{10}, e_{C}, e_{A}\right)$ in 35 developed countries, by sex, selected years 1970-2010 


\section{Acknowledgements}

The authors are thankful to Krystyna Kupiszewska for the language editing of the manuscript.

\section{Funding}

The study was supported by the National Science Center of Poland, grant no. 2012/05/B/HS4/00550 entitled "Measures of differences in mortality distributions". Fanny Janssen acknowledges support from the Netherlands Organisation for Scientific Research (NWO) (grant no. 452-13-001).

\section{Authors' contributions}

MM designed the study, collected the data, developed the formulas, performed the analyses and wrote the first draft of the manuscript. FJ contributed to the design of the study and wrote the final version of the manuscript. Both authors read and approved the final manuscript.

\section{Competing interests}

The authors declare that they have no competing interests.

\section{Author details}

${ }^{1}$ Warsaw School of Economics, Warsaw, Poland. ${ }^{2}$ Population Research Centre, University of Groningen, Groningen, The Netherlands. ${ }^{3}$ Netherlands Interdisciplinary Demographic Institute, The Hague, The Netherlands.

Received: 7 March 2016 Accepted: 8 July 2016

\section{Published online: 12 October 2016}

\section{References}

Asada, Y. (2006). Is health inequality across individuals of moral concern? Health Care Analysis, 14, 25-36.

Atkinson, A. B. (1970). On the measurement of inequality. Journal of Economic Theory, 2(3), 244-263.

Berrebi, Z.M., Silber. J. (1987). Deprivation, the Gini Index of Inequality and the Flatness of an Income Distribution. Mathematical Social Sciences, 18, 229-237.

Canudas-Romo, V. (2010). Three measures of longevity: Time trends and record values. Demography, 47, 299-312.

Cheung, S., Robine, J.-M., Tu, E., \& Caselli, G. (2005). Three dimensions of the survival curve: horizontalization, verticalization, and longevity extension. Demography, 42, 243-258.

d'Albis, H., Esso, L. J., \& Arolas, H. P. (2014). Persistent differences in mortality patterns across industrialized countries Plos one, 9(9), e106176.

Edwards, R. D. (2011). Changes in world inequality in length of life: 1970-2000. Population and Development Review 37(3), 499-528.

Edwards, R. D., \& Tuljapurkar, S. (2005). Inequality in life spans and a new perspective on mortality convergence across industrialized countries. Population and Development Review, 31, 645-674.

Edwards, R. D. (2013). The cost of uncertain life span. Journal of population economics, 26(4), 1485-1522.

Goesling, B., \& Firebaugh, G. (2004). The trend in international health inequality. Population and Development Review, 30(1), 131-146.

Human Mortality Database. (2014). University of California, Berkeley (USA), and Max Planck Institute for Demographic Research (Germany). Available at www.mortality.org (data downloaded on 12.10.2014) Accessed 12 Oct 2014

Janssen, F., Rousson, V., \& Paccaud, F. (2015). The role of smoking in changes in the survival curve: an empirical study in 10 European countries. Annals of Epidemiology, 25(4), 243-249.

Kolm, S.-C. (1976a). Unequal inequalities I. Journal of Economic Theory, 12(3), 416-442.

Kolm, S.C. (1976b). Unequal inequalities II. Journal of Economic Theory, 13(1), 82-111.

Kunst, A. E., Bos, V., Andersen, O., Cardano, M., Costa, G., Harding, S., Hemström, O., Layte, R., Regidor, E., Reid, A., Santana, P., Valkonen, T., \& Mo, J. P. (2004). Monitoring of trends in socioeconomic inequalities in mortality: experiences from a European project. Demographic Research, 2(4), 229-253. Special Collection.

Lexis, W. (1878). Sur la duree normale de la vie humaine et sur la theorie de la stabilite des rapports statistiques. Annales de Demographie Internationale, 2, 447-460.

Mackenbach, J. P., Bos, V., Andersen, O., Cardano, N., Costa, G., Harding, S., Reid, A., Hemström, O., Valkonen, T., \& Kunst, A. E. (2003). Widening socioeconomic inequalities in mortality in six Western European countries. International Journal of Epidemiology, 32, 830-837.

Mackenbach, J. P., Kunst, A. E., Cavelaars, A. E., Groenhof, F., \& Geurts, J. J. (1997). Socioeconomic inequalities in morbidity and mortality in Western Europe. The Lancet, 349, 1655-1659.

Moser, K., Shkolnikov, V., \& Leon, D. A. (2005). World mortality 1950-2000: divergence replaces convergence from the late 1980s. Bulletin of World Health Organization, 83, 202-209.

Oeppen, J., \& Vaupel, J. W. (2002). Broken limits to life expectancy. Science, 296, 1029-1031.

Silber, J. (1983). ELL (the Equivalent Length of Life) or another attempt at measuring development. World Development, 11(1), 21-29.

Silber, J. (1988). On inequality before death and life table summary measures. Genus, 44(1/2), 25-39.

Smits, J., \& Monden, C. (2009). Length of life inequality around the globe. Social Science and Medicine, 68(6), 1114-1123.

Thatcher, A. R., Cheung, S. L. K., Horiuchi, S., \& Robine, J.-M. (2010). The compression of deaths above the mode. Demographic Research, 22, 505-538.

Tuljapurkar, S., \& Edwards, R. D. (2011). Variance in death and its implications for modelling and forecasting mortality. Demographic Research, 24(21), 497-526.

Vaupel, J. W., Zhang, Z., \& van Raalte, A. A. (2011). Life expectancy and disparity: an international comparison of life table data. BMJ Open, 1(1), e000128-e000128

White, K. M. (2002). Longevity advances in high-income countries, 1955-96. Population and Development Review, 28, 59-76. Wilmoth, J. R., \& Horiuchi, S. (1999). Rectangularization revisited: variability of age at death within human populations. Demography, 36(4), 475-95. 